\title{
The globular cluster NGC 5286: color-magnitude diagram and variable stars
}

\author{
M. Zorotovic, ${ }^{1,2}$ M. Catelan, ${ }^{2}$ M. Zoccali, ${ }^{2}$ H. A. Smith ${ }^{3}$ \\ and B. J. Pritzl ${ }^{4}$ \\ ${ }^{1}$ ESO, Santiago, Chile \\ ${ }^{2}$ Pontifícia Universidad Católica, Santiago, Chile \\ ${ }^{3}$ Michigan State University, East Lansing, MI, USA \\ ${ }^{4}$ University of Wisconsin, Oshkosh, WI, USA
}

\begin{abstract}
We present $B V$ photometry and the results of a search for stellar variability in the globular cluster NGC 5286, which has tentatively been associated with the Canis Major dwarf spheroidal galaxy. Our results indicate an Oosterhoff type II for the cluster, which is unusual for an object of extragalactic origin.
\end{abstract}

Keywords. globular clusters: individual (NGC 5286), stars: variables: other

The full poster (in pdf format) is available at http://www . astro.iag.usp.br/ iaus266/Posters/pZorotovic.pdf. 